nacheinander ermordet. Die Suche nach den Tätern ist verwoben mit der Vorstellung von Protagonisten, die sich gegen rechte Gewalt wenden, teils mit Gegengewalt: Studenten, die in antifaschistischen Gruppen aktiv sind, sowie ein Freundeskreis beruflich etablierter Erwachsener, der sich als eine Art linke Bürgerwehr betätigt. Mit seinem „Drei Tote für Benni“ begibt sich Titus Simon, Professor für Jugendarbeit und Jugendhilfeplanung in Magdeburg, erstmals genau in die Welten, die er als Sozialarbeiter und späterer Hochschullehrer beackert hat. Seit fast dreißig Jahren befasst er sich beruflich mit dem Phänomen Rechtsextremismus. Das tut dem Krimi sehr gut, weil es der Handlung und den Figuren den notwendigen realistischen und beklemmenden Unterton verleiht. Simons Lieblingsermittler, Gotthilf Bröckle, der als Student im fortgeschrittenen Lebensalter in Magdeburg Vorlesungen besucht, gerät, ohne es zu forcieren, in die Ermittlungen und löst schließlich den Fall. Im Zuge seiner Nachforschungen wird klar, dass die üblichen Verdächtigen aus den verfeindeten politischen Milieus eben nicht die Täter sein können. Gut gelungen ist dabei das langsame Zusteuern auf den Täter, und die damit verbundene Wendung, die das Thema Umgang mit Rechtsextremismus beziehungsweise rechtsextrem orientierten Jugendlichen nochmals aus einem ganz anderen Blickwinkel beleuchtet. CHRISTINE SCHICK DOI 10.1007/s12054-010-0048-X

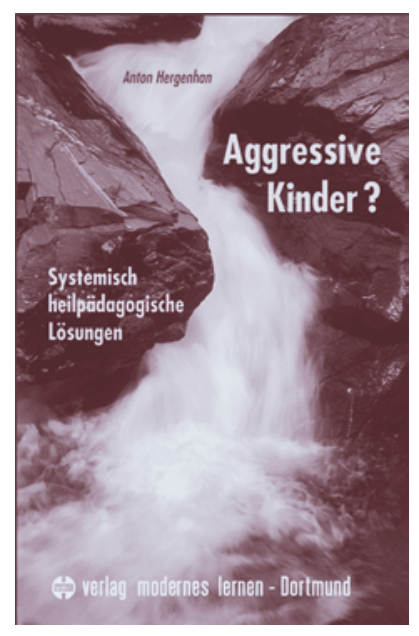

\section{ANTON HERGENHAN}

Aggressive Kinder

verlag modernes lernen 2010

ISBN 978-3-8080-0656-6

EURO 16,80

Der Autor des Buches „Aggressive Kinder“, Anton Hergenhan, ist als Psychologe in einer teilstationären Einrichtung für verhaltensauffällige Kinder tätig. Im Verlauf seiner langjährigen beruflichen Arbeit mit solchen Kindern hat er ein Konzept unter Nutzung verhaltenstherapeutischer, vor allem aber auch systemischer, Methoden entwickelt. Dieses Konzept, das in seiner Einrichtung erfolgreich und praktisch wirksam angewendet wird, stellt er in dem Buch vor. Damit widmet er sich einem aktuellen Problem der Pädagogik. Angesichts steigender verbaler und tätlicher Aggressionen unter Kindern ist die Frage, wie mit aggressiven Kindern umzugehen ist, damit ein friedlicheres Miteinander möglich wird, für Lehrer, Erzieher und Eltern zu einem wichtigen Thema geworden. Das Buch orientiert sich an der Praxis, am gelebten Alltag der Kinder und all derer, die mit ihnen umgehen, und gibt erprobte Anregungen für ein Leben miteinander, das Gewalt ausschließt. In der praktischen Arbeit mit verhaltensauffälligen Kindern wurden vom Autor sechs basale Kriterien für systemisch heilpädagogisches Handeln entwickelt, die im Buch anschaulich und nachvollziehbar dargestellt werden. Diesen Kriterien vorangestellt wird ein Leitsatz, der im Buch mehrfach auftaucht und den Rahmen für die Anwendung der Kriterien darstellt: „Das Kind hat Anspruch auf Führung und Information über seine Welt, es ist keine Erwachsener" behauptet Hergenhan und ist damit im Einklang mit den Kollegen seiner Einrichtung. Davon ausgehend werden die Kriterien entwickelt, in denen sich systemische Grundhaltungen unschwer wieder finden lassen: 1. Persönliche Präsenz; 2. Führung, die das Kind mündig sein lässt und respektiert; 3. Ausdrückliche Identifikation der Ressourcen, der Fähigkeiten; 4. Positive Beleuchtung des Symptoms; 5. Lösungsentwurf des Kindes; 6. Einbau des kindlichen Bezugssystems. Diese Kriterien werden eingehend beleuchtet, auf die systemische Theorie hin reflektiert und an praktischen Beispielen beschrieben. Systemische Ansätze werden konkret am Beispiel vorgestellt, ohne vorherige lange theoretische Abhandlungen. Das ist angenehm und hilfreich für den praktisch orientierten Leser, der sein eigenes Handlungsfeld erweitern will. Der theoretische Hintergrund wird jedoch vom Autor nicht vernachlässigt: Über Fußnoten verweist es auf zusätzliche Erklärungen, Hintergründe und Literatur.

Diese basalenHandlungskriterien, so Hergenhan, erleichtern den Pädagogen die Transparenz, Nachvollziehbarkeit und Reflexion ihrer eigenen Arbeit. Beim Lesen wird sehr schnell deutlich, welches Potenzial sich in der konsequenten Anwendung dieser Kriterien verbirgt, um ein Miteinander der Kinder untereinander, aber auch in Bezug auf Erwachsene und Kinder, friedlicher und konstruktiver zu gestalten. Einen wesentlichen Teil des Buches nimmt die Beschreibung einer Konfliktklärung in der Einrichtung Hergenhans ein. Dort kommen die Kinder selbst zu Wort, aber auch die systemisch arbeitenden Pädagogen. Ergänzend dazu ist ein ausführlicher Kommentar des Autors zum methodischen Vorgehen eingefügt. Die Umsetzung der sechs Kriterien im therapeutischen Gespräch wird sehr lebendig und anschaulich nachvollziehbar und lädt direkt zur Übernahme in eigenes pädagogisches und elterliches Handeln ein. Es erschließt sich dem Leser, durch welches genaue Vorgehen Frieden und Gewaltabbau im Gespräch mit den betroffenen Kindern erreicht werden kann und wie bereitwillig Kinder darauf eingehen, wenn ihnen gewaltfreie, sie respektierende, Lösungsmöglichkeiten für ihre Konflikte vorgelebt und nahe gebracht werden. Ein empfehlenswertes Buch für den praktischen pädagogischen Alltag mit verhaltensauffälligen Kindern, aber auch mit Kindern überhaupt. 